\title{
Prevalence of Vitamin D Deficiency Among Infants in Northern India: A Hospital Based Prospective Study
}

Swathi Chacham ${ }^{1}$, Swati Rajput ${ }^{2}$, Shilpa Gurnurkar ${ }^{3}$, Anissa Mirza ${ }^{2}$, Vartika Saxena ${ }^{4}$, Senkadhirdasan Dakshinamurthy ${ }^{4}$, Jaya Chaturvedi ${ }^{5}$, Jagdish P. Goyal ${ }^{6}$, Madhuradhar Chegondi ${ }^{7}$

1. Pediatrics, All India Institute of Medical Sciences, Rishikesh, IND 2. Biochemistry, All India Institute of Medical Sciences, Rishikesh, IND 3. Pediatric Endocrinology, Nemours Children's Hospital, Orlando, USA 4. Community and Family Medicine, All India Institute of Medical Sciences, Rishikesh, IND 5. Obstetrics and Gynaecology, All India Institute of Medical Sciences, Rishikesh, IND 6. Pediatrics, All India Institute of Medical Sciences, Jodhpur, IND 7. Pediatrics, University of Iowa Stead Family Children's Hospital, Iowa City, USA

Corresponding author: Madhuradhar Chegondi, madhuradhar-chegondi@uiowa.edu

\section{Abstract \\ Background: Vitamin D deficiency is one of the major nutritional deficiencies and an important contributor to nutritional and growth failure in infants, especially in those with low socioeconomic status.}

Aim: The primary objective of this study was to determine the proportion of vitamin D deficiency in infants, and the secondary objective was to assess the correlation between infant and maternal vitamin D levels.

Methods: This prospective, observational study was carried out at a tertiary care center, All India Institute of Medical Sciences in Rishikesh, Uttarakhand, India, in the Department of Pediatrics from January 2017 to December 2018. Children aged less than one year and their mothers were enrolled in the study. All the infants attending the Department of Pediatrics for well-child visits and sick-child visits were enrolled after obtaining written, informed consent. Infants with major congenital malformations and liver and kidney dysfunction were excluded. Serum vitamin $\mathrm{D}$ level of $<20 \mathrm{ng} / \mathrm{mL}$ was defined as vitamin $\mathrm{D}$ deficiency.

Results: A total of 200 infants and 200 mothers were enrolled in the study. Among the study infants, $80 \%$ were neonates, and $20 \%$ were infants beyond the neonatal period. The prevalence of vitamin $\mathrm{D}$ deficiency was $74 \%$ in infants and $85.5 \%$ in mothers. Nearly half of the infants and mothers had severe vitamin D deficiency. Logistic regression analysis showed a positive correlation between maternal and infant vitamin D levels $(\mathrm{r}=0.074, \mathrm{p}<0.001)$ and also with neonatal age group and low socioeconomic status. Hyperphosphatemia and hypocalcemia were predominant biochemical manifestations.

Review began 10/13/2020 Review ended 10/27/2020 Published 11/05/2020

\section{(c) Copyright 2020}

Chacham et al. This is an open access article distributed under the terms of the Creative Commons Attribution License CC-BY 4.0, which permits unrestricted use, distribution, and reproduction in any medium, provided the original author and source are credited.
Conclusion: The prevalence of vitamin D deficiency among the study infants was $74 \%$. Neonatal age group, lower socioeconomic status, and maternal vitamin D deficiency were major determinants of vitamin D deficiency in infants.

Categories: Endocrinology/Diabetes/Metabolism, Pediatrics, Epidemiology/Public Health

Keywords: infants, hypocalcemia, neonates, vitamin-d deficiency, maternal

\section{Introduction}

Vitamin D deficiency is one of the important nutritional deficiencies in children worldwide [1]. It was reported to occur among $96 \%$ of infants and $84 \%$ of pregnant women by the International Osteoporosis Foundation (IOF) in 2009 [2]. Vitamin D plays a key role in calcium absorption, bone mineralization, and aids in phosphate and magnesium metabolism [1]. The US Endocrine Society defined vitamin D deficiency as serum 25(OH) vitamin D levels less than $20 \mathrm{ng} / \mathrm{mL}$ and vitamin D insufficiency as serum 25(OH) vitamin D levels between 21 to $29.9 \mathrm{ng} / \mathrm{mL}$ [3]. Globally, it is estimated that around one billion people belonging to all age groups have vitamin D deficiency or insufficiency. In India, 50-90\% of children suffer from vitamin D deficiency or insufficiency predominantly due to lower dietary intake of calcium coupled with dark skin color and lack of adequate exposure to sunlight $[4,5]$.

Exclusive breastfeeding in the setting of maternal vitamin D deficiency, reduced dietary consumption, and decreased exposure to sunlight due to seasonal variation are leading causes of vitamin D deficiency among infants [4]. In severe cases, it manifests as rickets, characterized by frontal bossing, widening of wrists, bowing of legs, spontaneous fractures in infants, and can also lead to bony deformities, short stature, muscle weakness, and muscle pain in older children. Apart from skeletal manifestations, vitamin D deficiency can also present with growth failure, recurrent respiratory infections, and developmental delay [4]. Infants with malabsorption syndrome, cystic fibrosis, and nephrotic syndrome are at increased risk [4]. 
The key biochemical changes in infants with vitamin D deficiency consist of hypocalcemia,

hyperphosphatemia, and secondary hyperparathyroidism [4,5]. Recommended treatment for neonates with vitamin D deficiency includes 400-1000 international units (IU) of daily vitamin D for 8-12 weeks. Likewise, infants beyond the neonatal period require 1000-5000 IU/day of vitamin D for 8-12 weeks. Timely detection and treatment of vitamin D deficiency are crucial to prevent bony deformities and curtail respiratory morbidity [5]. The paucity of data on vitamin D deficiency status in neonates and infants from Uttarakhand, a northern state of India, prompted the present study.

\section{Materials And Methods}

This prospective, observational study was carried out at a tertiary care center, All India Institute of Medical Sciences in Rishikesh, Uttarakhand, India, in the Department of Pediatrics from 2017 to 2018. All the consecutive infants (aged $\leqslant 1$ year) who came to pediatric outpatient and inpatient units for sick- or wellchild visits were enrolled after obtaining written, informed consent. Infants with major congenital malformations, liver and kidney dysfunction, malabsorption syndrome, and those on tube feeding were excluded. Serum vitamin D level of $<20 \mathrm{ng} / \mathrm{mL}$ was defined as vitamin D deficiency [2]. All the study infants were clinically evaluated by using a predesigned, pretested proforma. Each infant's anthropometry, general physical examination findings, alongside clinical signs of hypocalcemia (jitteriness, tetany, etc.) and vitamin D deficiency (rachitic rosary, craniotabes, etc.), were monitored and recorded. Maternal details comprising socioeconomic status were recorded. The modified Kuppuswamy scale was used to classify the socioeconomic status [6]. From the enrolled infants, $2 \mathrm{ml}$ of venous blood was collected and analyzed for calcium, phosphorus, alkaline phosphatase, and 25-(OH) vitamin D levels. In addition, serum 25-(OH) vitamin D levels were also estimated in all the mothers of the enrolled infants. Vitamin D levels were analyzed by the chemiluminescence method using Siemens Advia Centaur XP (Siemens Diagnostics, Tarrytown, NY, USA). The research study was approved by the institution's review board (IM/RC104/2016/29), and written informed consent was obtained from the parents before study enrollment.

\section{Sample size calculation}

Considering a $60 \%$ prevalence of vitamin D deficiency, $80 \%$ power, and an allowable error of seven, we required 196 infant-mother pairs. We enrolled 200 infant-mother pairs for the study.

\section{Statistical analysis}

Descriptive statistics were used for calculating percentages, proportion, mean (standard deviation (SD)), and median (inter-quartile range (IQR)). Means of continuous variables between groups were compared by Student's t-test. Correlation between variables was assessed by using Pearson's correlation coefficient. Binary logistic regression analysis was performed simultaneously for finding the determinants of vitamin D deficiency. A significance level of 5\% was used for all of the statistical tests. The data was analyzed using Statistical Package for Social Sciences (SPSS) Version 22.0 (IBM SPSS Statistics for Windows; IBM Corp., Armonk, NY, USA).

\section{Results}

A total of 200 infant-mother dyads were included in the study. There were more neonates than infants beyond the neonatal age in the study $(80 \%, n=160$ vs. $20 \%, n=40)$. The majority of infants were males $(57.5 \%$, $\mathrm{n}=115)$. Seventy four percent of the infants $(\mathrm{n}=148)$ had vitamin D levels below $20 \mathrm{ng} / \mathrm{mL}$ and $26 \%(\mathrm{n}=52)$ had vitamin D levels over $20 \mathrm{ng} / \mathrm{mL}$. Therefore, the prevalence of vitamin D deficiency among infants in our study was $74 \%$ (Figure 1 ). 


\section{Cureus}

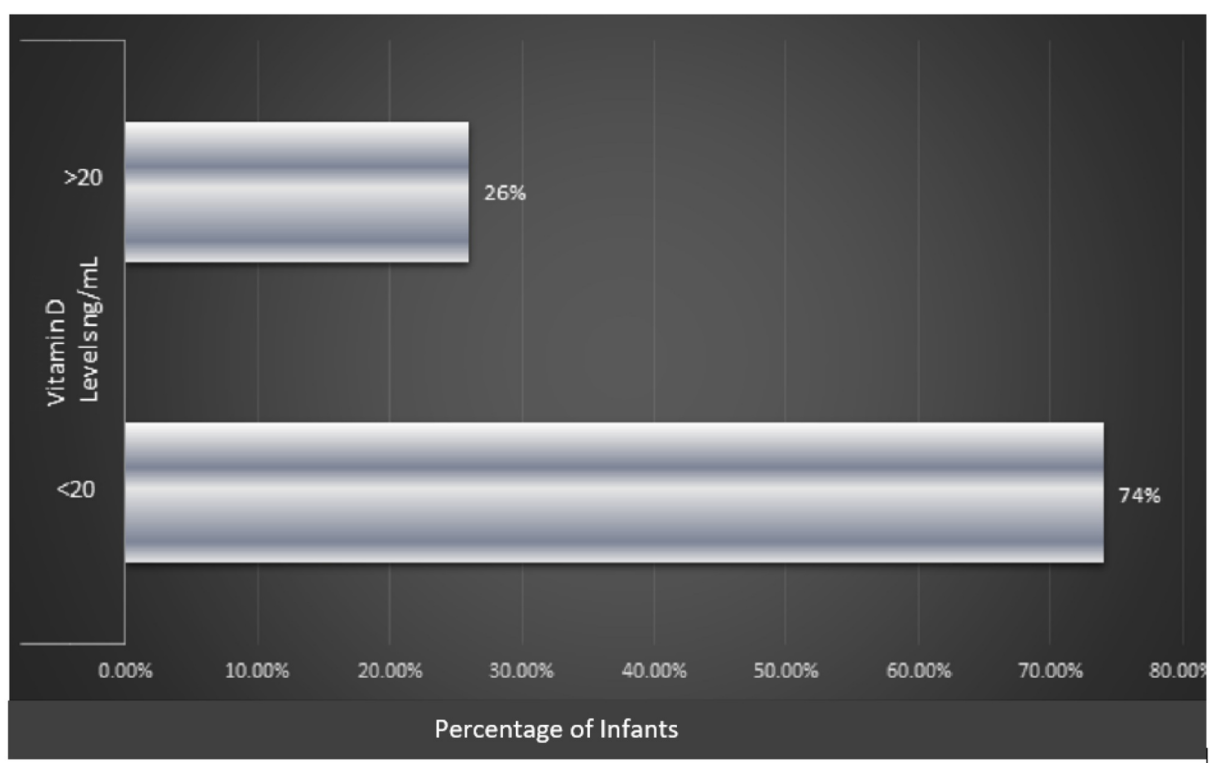

FIGURE 1: Prevalence of vitamin D deficiency among infants

Among those infants with vitamin D deficiency, $41.5 \%$ had severe vitamin D deficiency (vitamin D levels $<10$ $\mathrm{ng} / \mathrm{mL}$ ). When subgroup analysis was done, the prevalence of vitamin D deficiency was $79 \%$ in the neonatal age group (127/160), which was higher than the prevalence in those beyond the neonatal age group $(67.5 \%$, 27/40). Moreover, mean vitamin D levels were significantly lower in neonates than in the post-neonatal age group (p-value $<0.001$ ). All pertinent biochemical parameters are shown in Table 1 .

\begin{tabular}{|c|c|c|}
\hline Variables & Mean \pm SD & Median (IQR) \\
\hline Infant Vitamin D levels (ng/mL) & $16.81 \pm 12.50$ & $13(8-21.75)$ \\
\hline Calcium (mg/dL) & $8.96 \pm 1.65$ & $9(8-10)$ \\
\hline Phosphorus (mg/dL) & $11.98 \pm 6.68$ & $11(6-16)$ \\
\hline ALP (U/L) & $222.48 \pm 109.49$ & 203.5 (153.5 - 273.5) \\
\hline Mother Vitamin D levels (ng/mL) & $12.47 \pm 8.83$ & $10(7-15.75)$ \\
\hline
\end{tabular}

\section{TABLE 1: Mean and median values of biochemical parameters}

SD, standard deviation; IQR, interquartile range; ALP, alkaline phosphatase

About $14 \%(n=28)$ of children including neonates had vitamin $\mathrm{D}$ level between $20-30 \mathrm{ng} / \mathrm{mL}$ and $12 \%(\mathrm{n}=24)$ of them had level more than $30 \mathrm{ng} / \mathrm{mL}$. Only one-third of infants $(31 \%, \mathrm{n}=138)$ reported adequate sunlight exposure (15 to 30 minutes per day), and, interestingly, 108 infants among these 138 (78.2\%) had vitamin D deficiency. A major proportion of infants (85\%) were breastfed. Infants beyond the neonatal age group were analyzed for clinical features of vitamin D deficiency, and half of them had frontal bossing followed by craniotabes seen in $30 \%$ of infants. Likewise, rachitic rosary was seen in $10 \%$ of the infants. Other associated infant comorbidities comprised pneumonia (10\%) and developmental delay (5\%).

About $10.5 \%(\mathrm{n}=21)$ of the infants had a weight below the 3rd percentile. A sizable proportion of infants (25.8\%) had their length below the 3rd percentile, although most infants (74.2\%) had a length in the 3rd to 50th percentile. Seventy-five percent had an occipitofrontal circumference (OFC) in the 3rd to 50th percentile, and $23.7 \%$ had it over the 50 th percentile.

Looking at associated mineral abnormalities, the majority of the infants $(81.5 \%, \mathrm{n}=163)$ had hyperphosphatemia (serum phosphate more than $4.5 \mathrm{mg} / \mathrm{dL}$ ). Hypocalcemia (serum calcium less than 8.5 $\mathrm{mg} / \mathrm{dL}$ ) was found in $27 \%$ of infants. Alkaline phosphatase levels were normal (less than $400 \mathrm{U} / \mathrm{L}$ ) in the majority of infants (95.5\%), and only $4.5 \%(\mathrm{n}=9)$ had alkaline phosphatase levels more than $400 \mathrm{U} / \mathrm{L}$. However, among these nine infants, five (55.5\%) had vitamin D deficiency. Most infants were born at term 


\section{Cureus}

(89\%, $\mathrm{n}=178)$. Additionally, most infants were born appropriate for gestational age $(76.5 \%, \mathrm{n}=153)$. When the geographical area was taken into consideration, nearly one-third of the study infants $(36 \%, n=72)$ were from the hilly area. Ten infants in our study were on anti-epileptic drugs, and eight of them had vitamin D deficiency. Among the infants beyond the neonatal age group with vitamin D deficiency, 10\% had pneumonia, and 5\% had developmental delays.

In the maternal data, $85.5 \%(\mathrm{n}=171)$ of the mothers had vitamin $\mathrm{D}$ deficiency, of whom $54.5 \%$ had severe vitamin D deficiency. Only $14.5 \%$ of the mothers had vitamin D levels over $20 \mathrm{ng} / \mathrm{mL}$. Among the maternal comorbidities, pregnancy-induced hypertension was most frequent $(7.5 \%, \mathrm{n}=15)$. Adequate maternal sunlight exposure (15 to 30 minutes) was present in less than half $(47.5 \%, \mathrm{n}=95)$ of the study population. Among the 105 mothers without sunlight exposure, 69.5\% (n=73) had vitamin D deficiency.

Lower gestational age, lower socioeconomic status, and inadequate sunlight exposure in infants had a significant influence on mean vitamin $\mathrm{D}$ levels $(\mathrm{p}$-value=0.032). Birth weight also had a significant impact on vitamin D deficiency (80\% of infants with weight $<3$ rd percentile had vitamin D deficiency ( $\mathrm{p}$-value $<0.001$ ). Similarly, eight out of 10 infants with length $<3$ rd percentile had vitamin D deficiency (p-value $<0.001$ ). Vitamin D deficiency was similar among males and female infants. Breastfeeding versus formula feeding and total intake did not significantly influence the prevalence of vitamin D deficiency. Logistic regression analysis was utilized to predict the determinants of vitamin D deficiency (Table 2).

\begin{tabular}{|c|c|c|c|}
\hline Variable & $\beta$ coefficient & Adjusted Odds ratio & P-value \\
\hline Age & 2.3 & 10.1 & 0.001 \\
\hline Socio Economic status & 0.62 & 1.9 & 0.036 \\
\hline Mother Vitamin D status & 2.4 & 10.7 & 0.001 \\
\hline
\end{tabular}

\section{TABLE 2: Logistic regression analysis of determinants of vitamin D deficiency in infants}

Most families belonged to the lower middle class $(47.5 \%, \mathrm{n}=95)$, followed by the lower upper class $(33 \%$, $\mathrm{n}=66)$.

Neonatal age, low socioeconomic status, and maternal vitamin D deficiency were independently associated with a higher risk of vitamin D deficiency in infants. A hundred and forty-four infants (84.2\%) of 171 mothers with vitamin D deficiency also had vitamin D deficiency ( $p$-value $<0.001$ ). Likewise, there was a significant correlation (Pearson's correlation coefficient of 0.724 and p-value $<0.001$ ) between the infant's vitamin D levels and maternal vitamin D levels (Figure 2).

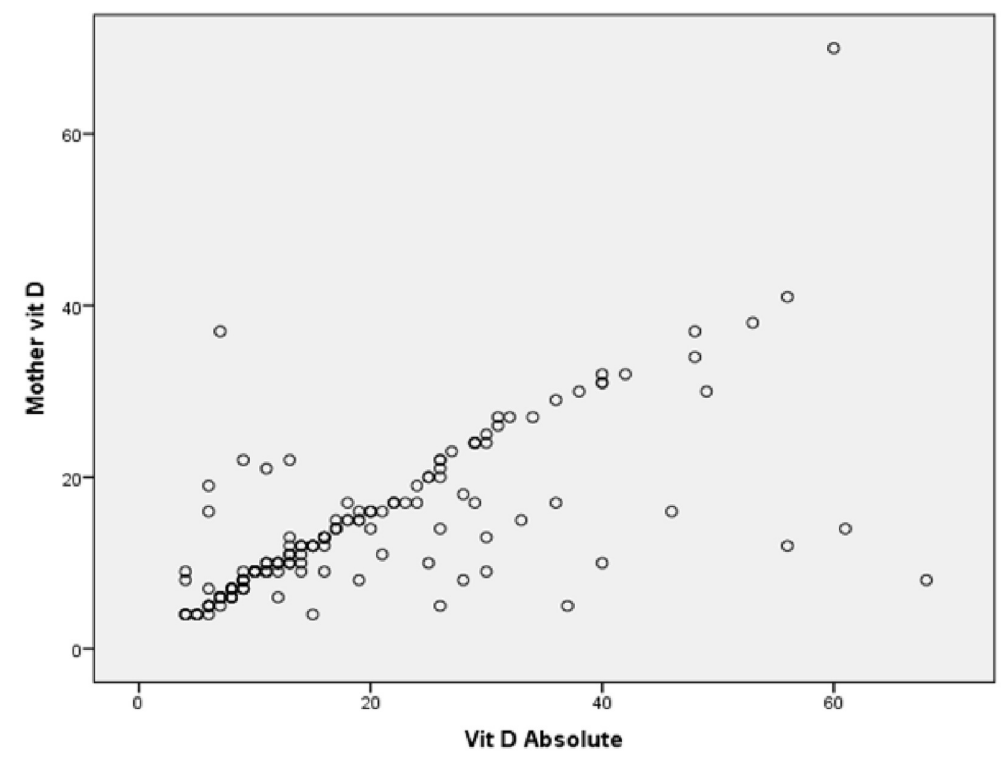

FIGURE 2: Correlation between maternal and infant vitamin D levels 


\section{Discussion}

The current study reports the prevalence of vitamin D deficiency in infants from Uttarakhand, a northern state in India. In 2009, the IOF found hypovitaminosis D in $96 \%$ of neonates, $91 \%$ of healthy school girls, and $84 \%$ of pregnant women in North India [2]. In India, $50-90 \%$ of the population suffers from vitamin D deficiency due to inadequate exposure to sunlight and a lower dietary intake $[4,5,7]$. Our study reported a $74 \%$ prevalence of vitamin D deficiency among infants and $85.5 \%$ among mothers, which is comparable to previous studies. The US Endocrine Society has classified vitamin D deficiency as $25(\mathrm{OH})$ vitamin D levels less than $20 \mathrm{ng} / \mathrm{mL}$, vitamin D insufficiency as levels $21-29 \mathrm{ng} / \mathrm{mL}$, sufficiency as levels more than $30 \mathrm{ng} / \mathrm{mL}$, and toxicity as vitamin D levels more than $150 \mathrm{ng} / \mathrm{mL}$ [3]. Similarly, the American Academy of Pediatrics (AAP, 2008) [8] and Institute of Medicine define vitamin D deficiency as serum 25(OH) vitamin D levels less than $15 \mathrm{ng} / \mathrm{mL}$, mild to moderate deficiency as $5-15 \mathrm{ng} / \mathrm{mL}$, severe deficiency as levels less than 5 $\mathrm{ng} / \mathrm{mL}$, and insufficiency as $16-20 \mathrm{ng} / \mathrm{mL}$. They define sufficiency as levels between $21-100 \mathrm{ng} / \mathrm{mL}$, excess as $101-149 \mathrm{ng} / \mathrm{mL}$, and intoxication as levels more than $150 \mathrm{ng} / \mathrm{mL}$ [9]. The Kidney Disease Outcome Quality Initiative seconds the AAP in defining vitamin D deficiency as levels $<15 \mathrm{ng} / \mathrm{mL}$ [10]. However, it defines insufficiency as levels between $16-30 \mathrm{ng} / \mathrm{mL}$ and sufficiency as levels of more than $30 \mathrm{ng} / \mathrm{mL}$. None of our study infants had vitamin D levels $>150 \mathrm{ng} / \mathrm{mL}$. The current study used the US Endocrine Society definition for defining vitamin D deficiency.

\section{Etiology}

Vitamin D levels are maintained by dietary intake and by cutaneous synthesis [11,12]. It was shown that half an hour exposure to sunlight in naked infants and two hours exposure for fully clothed babies can sustain weekly calcidiol levels of $11 \mathrm{ng} / \mathrm{mL}$ and, thus, prevent severe vitamin D deficiency [13]. Even a brief exposure to direct sunlight for 10 to 15 minutes can produce 10,000 to 20,000 IU of vitamin D. Various factors influence vitamin D synthesis from sunlight, such as latitude, pigmentation, and area of skin exposed [2]. Hence infants and children with darker skin pigmentation are at risk for decreased vitamin D synthesis. These infants require five to 10 times longer duration of sunlight exposure for the synthesis of equal levels of $25(\mathrm{OH})$ vitamin D in comparison to children with lighter pigmentation [14]. To maintain sufficient vitamin D levels, Asian children require three times greater sunlight exposure than white Americans due to darker skin color. The AAP recommends avoiding direct sunlight exposure in infants less than six months of age [15], which can add to vitamin D deficiency [16-18]. However, neonates and infants have enhanced ability to produce vitamin $D$ from sunlight when compared to adults due to higher surface area to volume ratio $[19,20]$.

In our study, we have shown that the infants' sunlight exposure had a significant impact on vitamin $\mathrm{D}$ deficiency. Infants belonging to lower socioeconomic status had a higher prevalence of vitamin D deficiency than those from a higher socioeconomic status. This could be explained by the fact that children from lower socioeconomic status are likely to have reduced calcium and vitamin D intake, which was ascertained in our study [18]. Preterm neonates are at greater risk for vitamin D deficiency due to reduced placental transfer, inadequate exposure to sunlight, and decreased vitamin D stores due to low-fat mass. Of the 18 preterm neonates in this study, 15 had vitamin D deficiency.

Almost four-fifths of neonates and two-thirds of infants beyond the neonatal age group had vitamin D deficiency. There was a higher prevalence of vitamin $D$ deficiency in neonates than in infants beyond the neonatal age group, which is similar to the IOF report [2]. The mean vitamin D levels were also significantly lower in neonates than in infants beyond the neonatal age group.

In our study, most infants with weight and height lower than the 3rd percentile had vitamin D deficiency. This can be well explained by lower nutrient intake, especially of calcium and vitamin D. Also, growth failure can be a manifestation of vitamin D deficiency [13]. Maternal vitamin D deficiency was shown to be an important risk factor for infants with vitamin D deficiency, which is similar to the findings reported by the IOF [2].

Predominant biochemical changes in infants with vitamin D deficiency included hyperphosphatemia, hypocalcemia, and hyperparathyroidism. This can be explained by immature renal tubular excretion in neonates, who were the predominant age group. Also, lower glomerular filtration rate, low intact parathyroid hormone (iPTH) levels, and renal tubular unresponsiveness to PTH, especially in the first three days of life, could have led to hyperphosphatemia alongside vitamin D deficiency. The coexisting metabolic bone disease, particularly among preterm small-for-gestational-age (SGA) neonates, could have contributed further to these biochemical changes. Alkaline phosphatase levels were normal (less than $400 \mathrm{U} / \mathrm{L}$ ) in most of the infants from our study, and only $4.5 \%$ had elevated alkaline phosphatase levels of more than $400 \mathrm{U} / \mathrm{L}$. This could be explained by the fact that during the neonatal period, the alkaline phosphatase levels do not change much $[21,22]$.

Breast milk is a poor source of vitamin D (average 22 units/L; range 15-50 units/L) even in a vitamin D sufficient mother. Vitamin D supplementation of lactating mothers with 3000-6000 IU enhances the antirachitic effect of breast milk without leading to maternal hypervitaminosis D. Even in the current study, a major proportion of infants who were exclusively breastfed had a higher prevalence of vitamin $D$ deficiency. After dietary intake, vitamin D is metabolized to calcidiol in the liver by the cytochrome P450 
enzyme system. Medications such as carbamazepine, oxcarbazepine, phenobarbital, and phenytoin induce cytochrome P450 and enhance vitamin D metabolism and reduce the circulating active vitamin D concentrations $[3,4,18]$. These drugs can even cause hypocalcemia and precipitate seizures. Drugs such as corticosteroids and azole antifungals also affect the absorption, metabolism, or activation of vitamin D. As a very small proportion of the study infants and mothers were on anti-epileptic drugs, their influence on the prevalence of vitamin D deficiency could not be assessed.

\section{Clinical features and morbidities}

Vitamin D deficiency can go unnoticed without many clinical manifestations in the early phase and progress to florid rickets if left undiagnosed in older children. However, there may not be any clinical manifestations, especially skeletal changes in the neonatal age group, apart from the features of hypocalcemia [13]. In this study, $27 \%$ of the infants with vitamin D deficiency had hypocalcemia, and $10 \%$ had jitteriness. Vitamin D plays an important role in preventing respiratory infections [4], and 10\% of the study infants beyond the neonatal age group had pneumonia along with vitamin D deficiency. Also, 5\% had developmental delays. Frontal bossing, craniotabes, and rachitic rosary are important clinical manifestations of vitamin D deficiency in older infants and children [13], and these were seen in 50\%, 30\%, and 10\% respectively, of the study infants who were beyond the neonatal age group. Our study ascertains exclusive breastfeeding and poor sunlight exposure as etiological factors for vitamin D deficiency. Likewise, the neonatal age group, low socioeconomic status, and maternal vitamin D deficiency were major independent determinants for Vitamin D deficiency in infants, as reported in previous studies $[23,24]$.

Vitamin D deficiency is treated with vitamin D supplementation, either orally or intramuscular, along with adequate calcium supplementation to prevent hungry bone syndrome, which occurs due to underlying hypocalcemia and remineralization of the bone matrix. All the neonates and infants with vitamin D deficiency in our study were supplemented with calcium and vitamin D as per age-appropriate protocols. There are three oral forms of vitamin D, namely, ergocalciferol (25-hydroxyvitamin D2 or vitamin D2), cholecalciferol (25-hydroxyvitamin D3 or vitamin D3), and calcitriol (1,25(OH)2D). It is preferred to use vitamin D2 and D3 in infants and young children [18,25]. Calcitriol poses the risk of cholelithiasis and hypercalcemia and hence should be avoided as the first-line agent. It was reported that vitamin D3 has better efficacy in increasing serum $25(\mathrm{OH}) \mathrm{D}$ concentration (three times) than vitamin D2. Vitamin D2 or D3 is given at a dose of 1,000 IU daily in neonates, 1,000-5,000 IU daily for 12 weeks in infants, and up to 5000 IU daily in children older than one year, to achieve a total cumulative dose of 200,000 to 600,000 IU [4]. Children with malabsorption syndromes and those receiving anticonvulsants, glucocorticoids, antifungals, or antiretroviral medications need higher $(1500 \mathrm{IU})$ and prolonged oral doses of vitamin D to achieve optimal serum levels $[4,13,18]$.

\section{Prevention}

As found in this study, maternal vitamin D status has a significant impact on the infant's vitamin D status. It is advised to assess $25(\mathrm{OH}) \mathrm{D}$ levels in all pregnant women and treat vitamin D deficiency with 3000-6000 IU of vitamin D3 until adequate serum levels of 25(OH)D (levels >20 ng/mL) are achieved [26-28]. Preterm infants require 400-800 IU of D3 per day from birth due to insufficient placental transfer of maternal vitamin $\mathrm{D}$ and other comorbidities associated with prematurity that may lead to a decreased intake or absorption of vitamin D [29]. In this study, all the mothers with vitamin D deficiency were also supplemented with vitamin $\mathrm{D}$ and calcium as per the unit protocol.

\section{Strengths}

Our study included a large sample size of mother-infant dyads. Extensive clinical assessment and biochemical workup was performed. This study aided in the early detection of infants with vitamin D deficiency and thus curtailed morbidity related to it. Detection and treatment of maternal vitamin D deficiency could prevent subsequent neonatal vitamin D deficiency.

\section{Limitations}

This is a single-center study and included only infants. The prevalence of vitamin D deficiency may vary depending on the region, and caution should be exercised in generalizing the study results.

\section{Conclusions}

In our large group infant-mother dyads, the prevalence of vitamin D deficiency was significantly high. Neonatal age group, low socioeconomic status, and maternal vitamin D deficiency were independent determinants of vitamin D deficiency in infants. This high prevalence of vitamin D deficiency among infants in our region necessitates adequate nutritional support and supplementation for mothers and children to prevent morbidity associated with vitamin D deficiency.

\section{Additional Information}

\section{Disclosures}


Human subjects: Consent was obtained by all participants in this study. Institutional Ethics Committee (IEC), All India Institute of Medical Sciences. Rishikesh, Uttarakhand issued approval IM/RC104/2016/29. Animal subjects: All authors have confirmed that this study did not involve animal subjects or tissue. Conflicts of interest: In compliance with the ICMJE uniform disclosure form, all authors declare the following: Payment/services info: All authors have declared that no financial support was received from any organization for the submitted work. Financial relationships: All authors have declared that they have no financial relationships at present or within the previous three years with any organizations that might have an interest in the submitted work. Other relationships: All authors have declared that there are no other relationships or activities that could appear to have influenced the submitted work.

\section{Acknowledgements}

We thank the All India Institute of Medical Sciences, Rishikesh, for providing the intramural grant for this study.

\section{References}

1. Holick MF: Vitamin D: extraskeletal health. Rheum Dis Clin North Am. 2012, 38:141-160. 10.1016/j.rdc.2012.03.013

2. Mithal A, Wahl DA, Bonjour JP, et al.: Global vitamin D status and determinants of hypovitaminosis D . Osteoporos Int. 2009, 20:1807-1820. 10.1007/s00198-009-0954-6

3. Holick MF, Binkley NC, Bischoff-Ferrari HA, et al.: Evaluation, treatment, and prevention of vitamin D deficiency: an Endocrine Society clinical practice guideline. J Clin Endocrinol Metab. 2011, 96:1911-1930. 10.1210/jc.2011-0385

4. Balasubramanian S, Dhanalakshmi K, Amperayani S: Vitamin D deficiency in childhood-a review of current guidelines on diagnosis and management. Indian Pediatr. 2013, 50:669-675. 10.1007/s13312-013-0200-3

5. Joiner TA, Foster C, Shope T: The many faces of vitamin D deficiency rickets . Pediatr Rev. 2000, 21:296-302. 10.1542/pir.21-9-296

6. Sharma R: Kuppuswamy's socioeconomic status scale-revision for 2011 and formula for real-time updating . Indian J Pediatr. 2012, 79:961-962. 10.1007/s12098-011-0679-3

7. Harinarayan CV, Joshi SR: Vitamin D status in India--its implications and remedial measures . J Assoc Physicians India. 2009, 57:40-48.

8. Gartner LM, Greer FR; Section on Breastfeeding and Committee on Nutrition: Prevention of rickets and vitamin D deficiency: new guidelines for vitamin D intake. Pediatrics. 2003, 111:908-910. 10.1542/peds.111.4.908

9. Institute of Medicine: Dietary Reference Intakes for Calcium and Vitamin D . Ross AC, Taylor CL, Yaktine AL, Del Valle HB (ed): The National Academies Press, Washington, DC; 2011. 10.17226/13050

10. National Kidney Foundation, Inc. Guideline 8. Prevention and treatment of vitamin D insufficiency and vitamin D deficiency in CKD patients. KDOQI clinical practice guidelines for bone metabolism and disease in children with chronic kidney disease. (2005). http://kidneyfoundation.cachefly.net/professionals/KDOQI/guidelines_pedbone/guide8.htm.

11. Thacher TD, Fischer PR, Pettifor JM, Lawson JO, Isichei CO, Reading JC, Chan GM: A comparison of calcium, vitamin D, or both for nutritional rickets in Nigerian children. N Engl J Med. 1999, 341:563-568. 10.1056/NEJM199908193410803

12. Pettifor JM: Nutritional rickets: deficiency of vitamin D, calcium, or both? . Am J Clin Nutr. 2004, 80:1725S1729S. 10.1093/ajcn/80.6.1725S

13. Casey CF, Slawson DC, Neal LR: Vitamin D supplementation in infants, children, and adolescents . Am Fam Physician. 2010, 81:745-748.

14. Clemens TL, Henderson SL, Adams JS, Holick MF: Increased skin pigment reduces the capacity of skin to synthesise vitamin D3. Lancet. 1982, 319:74-76. 10.1016/s0140-6736(82)90214-8

15. Committee on Environmental Health: Ultraviolet light: a hazard to children. Pediatrics. 1999, 104:328-333. 10.1542/peds.104.2.328

16. Reichrath J: The challenge resulting from positive and negative effects of sunlight: how much solar UV exposure is appropriate to balance between risks of vitamin D deficiency and skin cancer?. Prog Biophys Mol Biol. 2006, 92:9-16. 10.1016/j.pbiomolbio.2006.02.010

17. Lucas RM, Ponsonby AL: Considering the potential benefits as well as adverse effects of sun exposure: can all the potential benefits be provided by oral vitamin D supplementation?. Prog Biophys Mol Biol. 2006, 92:140-149. 10.1016/j.pbiomolbio.2006.02.019

18. Misra M, Pacaud D, Petryk A, Collett-Solberg PF, Kappy M; Drug and Therapeutics Committee of the Lawson Wilkins Pediatric Endocrine Society: Vitamin D deficiency in children and its management: review of current knowledge and recommendations. Pediatrics. 2008, 122:398-417. 10.1542/peds.2007-1894

19. Chacham S, Pasi R, Chegondi M, Ahmad N, Mohanty SB: Metabolic bone disease in premature neonates- an unmet challenge (Epub ahead of print). J Clin Res Pediatr Endocrinol. 2019, 10.4274/jcrpe.galenos.2019.2019.0091

20. Abrams SA; Committee on Nutrition: Calcium and vitamin D requirements of enterally fed preterm infants . Pediatrics. 2013, 131:e1676-e1683. 10.1542/peds.2013-0420

21. Hill TR, Flynn A, Kiely M, Cashman KD: Prevalence of suboptimal vitamin D status in young, adult and elderly Irish subjects. Ir Med J. 2006, 99:48-49.

22. Taylor JA, Richter M, Done S, Feldman KW: The utility of alkaline phosphatase measurement as a screening test for rickets in breast-fed infants and toddlers: a study from the Puget Sound Pediatric Research Network. Clin Pediatr (Phila). 2010, 49:1103-1110. 10.1177/0009922810376993

23. Andiran N, Yordam N, Ozön A: Risk factors for vitamin D deficiency in breast-fed newborns and their mothers. Nutrition. 2002, 18:47-50. 10.1016/s0899-9007(01)00724-9 


\section{Cureus}

24. Fink C, Peters RL, Koplin JJ, Brown J, Allen KJ: Factors affecting vitamin D status in infants . Children (Basel). 2019, 6:7. 10.3390/children6010007

25. Munns C, Zacharin MR, Rodda CP, et al.: Prevention and treatment of infant and childhood vitamin D deficiency in Australia and New Zealand: a consensus statement. Med J Aust. 2006, 185:268-272. 10.5694/j.1326-5377.2006.tb00558.x

26. Holick MF: Vitamin D status: measurement, interpretation, and clinical application. Ann Epidemiol. 2009, 19:73-78. 10.1016/j.annepidem.2007.12.001

27. Gordon CM, Williams AL, Feldman HA, May J, Sinclair L, Vasquez A, Cox JE: Treatment of hypovitaminosis D in infants and toddlers. J Clin Endocrinol Metab. 2008, 93:2716-2721. 10.1210/jc.2007-2790

28. Soliman AT, El-Dabbagh M, Adel A, Al Ali M, Aziz Bedair EM, Elalaily RK: Clinical responses to a mega-dose of vitamin D3 in infants and toddlers with vitamin D deficiency rickets. J Trop Pediatr. 2010, 56:19-26. 10.1093/tropej/fmp040

29. Cho SY, Park HK, Lee HJ: Efficacy and safety of early supplementation with 800 IU of vitamin D in very preterm infants followed by underlying levels of vitamin D at birth. Ital J Pediatr. 2017, 43:45.

10.1186/s13052-017-0361-0 